\title{
Aicardi-Goutieres Syndrome Presenting with Congenital Glaucoma
}

\author{
Vykuntaraju K. Gowda ${ }^{1}$ (D) Hemadri Vegda ${ }^{1} \cdot$ Sanjay K. Shivappa ${ }^{2} \cdot$ Naveen Benakappa $^{2}$
}

Received: 11 November 2019 / Accepted: 10 January 2020 / Published online: 5 February 2020

(C) Dr. K C Chaudhuri Foundation 2020

To the Editor: Aicardi-Goutieres syndrome (AGS) is genetically determined encephalopathy [1]. The main features are progressive encephalopathy, failure to thrive, basal ganglia calcification, leukodystrophy, and cerebrospinal fluid (CSF) lymphocytosis, mimicking TORCH (Toxoplasmosis, Others, Rubella, Cytomegalovirus, Herpes) infections. Here we report a rare phenotype of AGS presenting as congenital glaucoma.

A 15-mo-old boy, born to consanguineously married couple with normal birth history presented with developmental delay and visual impairment since 4 mo of age. The child had achieved social smile and mother recognition by 3 mo of age. At 4 mo of age he lost eye to eye contact. Outside, the child was diagnosed as congenital glaucoma at 6 mo of age and underwent trabeculectomy at 1 year of age for both eyes. At 15 mo of age, the child had only partial head control, no eye to eye contact, no palmar grasp, and no cooing. The child had microcephaly, not following and fixing light, megalocornea and dystonia. CSF analysis showed 14 lymphocytes with normal sugar and protein. CT brain showed bilateral basal ganglia calcifications. MRI brain showed periventricular white matter signal changes. Family history of elder male sibling, who is currently $4 \mathrm{y}$ of age, also had congenital glaucoma, developmental delay and seizures. Elder child's MRI brain showed cystic changes in the temporal lobe with white matter signal changes. TORCH infections were ruled out, based on positive family history and negative serology. We suspected AGS and testing by targeted next generation sequencing revealed homozygous $R N A S E H 2 C$ gene pathogenic variant chr11:65487856G > A c.205C > T p.Arg69Trp. Confirmation was done by Sanger sequencing for index case, sibling and also for both parents. It was in homozygous state in elder sibling and heterozygous state in both parents.

Vykuntaraju K. Gowda

drknvraju08@gmail.com

1 Department of Pediatric Neurology, Indira Gandhi Institute of Child Health, Bengaluru, Karnataka 560029, India

2 Department of Pediatrics, Indira Gandhi Institute of Child Health, Bengaluru, Karnataka, India
We report two siblings with AGS who presented as global developmental delay and congenital glaucoma. AGS is a genetic encephalopathy which mimics TORCH infections [2]. This child was suspected to have AGS based on developmental delay, consanguineous marriage, family history of similar sibling, and cerebral calcifications. AGS has been divided into seven types based on 7 different genetic mutations [3]. As per our knowledge there are only two reports with congenital glaucoma: one report, with three children of two families, where genetic test was not done [4]; second case report of aniridia with glaucoma with IFIHI gene involvement [5]. This is the first case report from India with AGS presenting in two siblings with congenital glaucoma. AGS should be considered in TORCH like infections with familial recurrence and glaucoma.

\section{Compliance with Ethical Standards}

Conflict of Interest None.

\section{References}

1. Rice GI, del Toro DY, Jenkinson EM, et al. Gain-of-function mutation in IFIH1 cause a spectrum of human disease phenotype associated with upregulated type 1 interferon signaling. Nat Genet. 2014;46:503-9.

2. Rice GI, Patrick T, Parmar R, et al. Clinical and molecular phenotype of Aicardi Goutieres syndrome. Am J Hum Genet. 2007;81:P71325.

3. Crow YJ, Chase DS, Schmidt JL, et al. Characterization of human disease phenotypes associated with mutations in TREX1, RNASEH2A, RNASEH2B, RNASEH2C, SAMHD1, ADAR and IFIH1. Am J Hum Genet A. 2015;167:296-312.

4. Crow YJ, Massey RF, Innes JR, et al. Congenital glaucoma and brain stem atrophy as features of Aicardi Goutières syndrome. Am J Med Genet. 2004;129A:303-7.

5. Musalem HM, Dirar QS, A1-Hazzaa SAF, Al Zoba AA, ElMansoury J, et al. Unusual association of aniridia with AicardiGoutieres syndrome-related congenital glaucoma in a tertiary care centre. Am J Case Rep. 2018;19:500-4.

Publisher's Note Springer Nature remains neutral with regard to jurisdictional claims in published maps and institutional affiliations. 